\title{
Face Image Quality Evaluation for ISO/IEC Standards 19794-5 and 29794-5
}

\author{
Jitao Sang, Zhen Lei, and Stan Z. Li ${ }^{\star}$ \\ Center for Biometrics and Security Research, \\ Institute of Automation, Chinese Academy of Sciences, \\ 95 Zhongguancun Donglu, Beijing 100190, China \\ \{jtsang,zlei,szli\}@cbsr.ia.ac.cn
}

\begin{abstract}
Face recognition performance can be significantly influenced by face image quality. The approved ISO/IEC standard 19794-5 has specified recommendations for face photo taking for E-passport and related applications. Standardization of face image quality, ISO/IEC 29794-5, is in progress. Bad illumination, facial pose and out-of-focus are among main reasons that disqualify a face image sample. This paper presents several algorithms for face image quality assessment. Illumination conditions and facial pose are evaluated in terms of facial symmetry, and implemented based on Gabor wavelet features. Assessment of camera focus is done based on discrete cosine transform (DCT). These methods are validated by experiments.
\end{abstract}

Keywords: Face image quality, international standard, facial symmetry, out-of-focus.

\section{Introduction}

The approved ISO/IEC standard 19794-5[3] includes instructions for lighting, facial pose, focus, and so on, for taking face photos for applications such as Epassport. Illustrated by Fig 1, which is a standard face image, we can see besides normative requirements of size and proportion, the face is uniformly illuminated and captured from right ahead with no rotation or pitching, out-of-focus is not admissible either.

In ISO/IEC 19794-5, out-of-focus, non-frontal posture and side lighting are regarded as primary elements for poor face image quality. Clause 7.3.3 has regularization that captured image shall always be in focus from nose to ears and chin to crown, while clauses 7.2.2 and 7.2.7-7.2.10 require uniform illumination and fix the angel control for deviation from frontal head posture with respect to Pan/Tilt/Roll axis.

Face images of bad quality which do not accord with the requirements of the standards is a reason leading to face recognition performance degradation.

\footnotetext{
^ Corresponding author.
} 


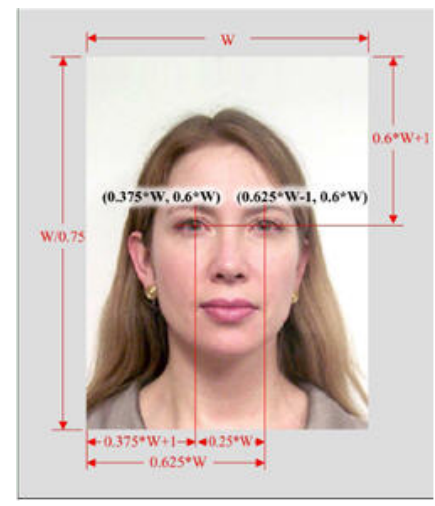

Fig. 1. Standard face image agreed with ISO/IEC 19794-5 3

Table 1. Face image requirements in ISO/IEC-19794-5

\begin{tabular}{|c|c|c|}
\hline Clause & Attribute & Constraint \\
\hline \multirow{4}{*}{ Scene } & Posture & control on deviation from frontal \\
\cline { 2 - 3 } & Illumination & Uniformly illuminated with no shadow \\
\cline { 2 - 3 } & Background & Plain light-colored \\
\cline { 2 - 3 } & Eyes & Open and clearly visible \\
\cline { 2 - 3 } & Glasses & No flash reflections, dark tint or heavy frames \\
\cline { 2 - 3 } & Mouth & Closed and clearly visible \\
\hline Photographic & Head position & Placed in the center \\
\cline { 2 - 3 } & Distance to camera & Moderate head size \\
\cline { 2 - 3 } & Color & Color neutral and no red eye \\
\cline { 2 - 3 } & Exposure & Appropriate brightness \\
\hline Digital & Focus & No out-of-focus and in Good sharpness \\
\cline { 2 - 3 } & Resolution & Width constraint of the head \\
\hline
\end{tabular}

Non-standard lighting or pose and out-of-focus are among the main reasons responsible for the performance degradation. One solution, where most researchers commit themselves, is to improve the algorithm itself by making it robust to possible degradation.

In virtue of face image quality evaluation method, face image quality are evaluated before subsequent enrollment and comparison and unqualified images will be abandoned so as to stabilize the systems performance. A framework for image quality evaluation was proposed in [1] and subsequently adopted in a draft of ISO/IEC 29794-5 2.

Draft of ISO/IEC 29794-5[2] specifies methodologies for computation of objective and quantitative quality scores for facial images. Approaches for the determination of certain aspects, such as facial symmetry, resolution and size, illumination intensity, brightness, contrast, color, exposure, sharpness, etc, are introduced. 
Moreover, draft of ISO/IEC 29794-5[2] suggests that facial quality be categorized into static subject and dynamic subject characteristics. In addition to the normative requirements presented in ISO/IEC 19794-5, open issue talking over objective metric for human perceived quality vs. metric for psFAR/psFRR are proposed in this draft, thus making it more practical and comprehensive. Measurements of face image quality are categorize into three aspects as scene requirements, photographic requirements and digital requirements, as in Table 1

In this paper, we present methods for face image quality evaluation. Gabor wavelets are used as basis features to estimate the symmetry and then to evaluate lighting and pose conditions. Discrete Cosine Transform (DCT) and Inverse DCT (IDCT) are used to calculate a degree of out-of-focus. Experiment results are shown to illustrate the methods.

\section{Gabor-Based Facial Symmetry}

The illumination and pose variations are two main issues that cause severe performance degradation for most existing systems [9]. Uneven lighting and deviation from frontal posture give rise to severe facial asymmetry. So we can use facial symmetry to evaluate quality degradations caused by non-frontal lighting and improper facial pose. Difference between the left and right half regions of face give a visualized description of facial asymmetry, where the difference is zero when the face is strictly symmetric.

Suggested by the definition, difference between one face image and its mirror is a natural way to measure the symmetry. However, this method is too sensitive to face alignment which means it is available only when geometric centerline of the face image matches very well with the physical centerline.

Local filters which is more robust to alignment while sensitive to posture and illumination are needed. Gabor wavelets, Ordinal and Local Binary Patterns(LBP) filters provide effective local features met these requirements. Graft of ISO/IEC 29794-5[2] introduces a LBP-based method. LBP is sort of coding based on comparison with adjacent areas, thus will fail in extreme case when surrounding pixels brighten or darken in the same level. In this paper we propose to employ imaginary of Gabor filters as a solution to assess the facial symmetry.

Gabor feature is a popular descriptor in face recognition [4]. The Gabor kernels are defined as follows:

$$
\psi_{\mu, \nu}=\frac{k_{\mu, \nu}^{2}}{\sigma^{2}} \exp \left(-\frac{k_{\mu, \nu}^{2} z^{2}}{2 \sigma^{2}}\right)\left[\exp \left(i k_{\mu, \nu} z\right)-\exp \left(-\frac{\sigma^{2}}{2}\right)\right]
$$

where $\mu$ and $\nu$ define the orientation and scale of the Gabor kernels respectively, $z=(x, y)$, and the wave vector $k_{\mu, \nu}$ is defined as follows:

$$
k_{\mu, \nu}=k_{\nu} e^{i \phi_{\mu}}
$$

where $k_{\nu}=k_{\max } / f^{\nu}, k_{\max }=\pi / 2, f=\sqrt{2}, \phi_{\mu}=\pi \mu / 8$.

In face recognition, the magnitude of Gabor feature is usually adopted due to its robustness to illumination and expression. However, in this case, we require 

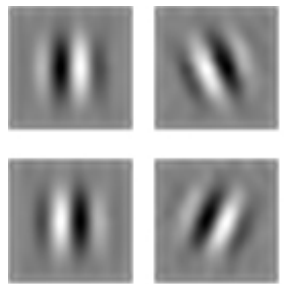

$0^{\prime}$

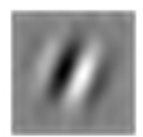

$22.5^{\prime}$
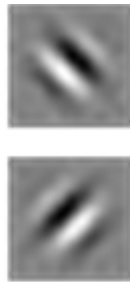

$45^{\prime}$
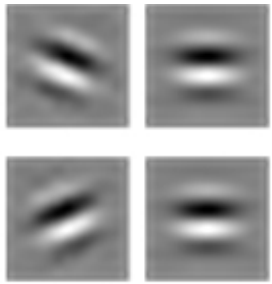

$67.5^{\prime}$

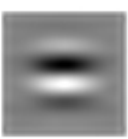

$90^{\prime}$

Fig. 2. Gabor filters of 5 orientations (the second row is the mirror filter corresponding to the first)
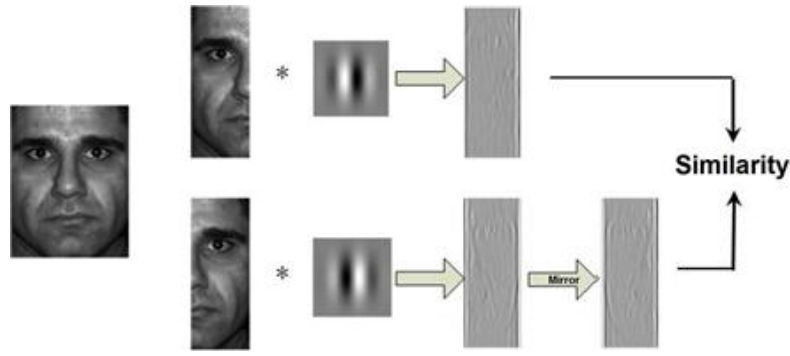

Fig. 3. Evaluation of facial symmetry using Gabor filters

the feature that is sensitive to illumination and pose. Therefore, the imaginary of Gabor filters, which not only has the characteristic of gradient operator, but also has orientation selectivity and stability, is utilized and believed to be competent for asymmetric. Fig. 2 shows the imaginary of Gabor filters in five orientations selected in this paper and Fig. 3illustrates the whole process for face asymmetric measurement. Firstly, five pairs of mirror symmetric Gabor filters are acted on left and right half regions of a face image respectively. Secondly, the asymmetry is measured as the difference between the corresponding Gabor responses on left and right part calculated as:

$$
\text { Asymmetry }=\sum_{i=1}^{M} \sum_{j=1}^{N}|L(i, j)-R(i, j)|
$$

where $M$ and $N$ is the height and width of a image, $L$ and $R$ indicates the left and right half part of Gabor responses. Obviously, the bigger the value of Asymmetry, the worse the facial symmetry.

\section{DCT-Based Sharpness}

Most optical systems are equipped with components of automated out-of-focus detection and blur adjustment [6]. Blurriness, resulting from out-of-focus, is 
treated as important measurement for image quality evaluation[5]. In view of image processing, out-of-focus leads to lost in high-spatial frequency. Thus we proposed to use DCT to evaluate it on frequency domain.

Sharpness is another crucial factor affecting the performance of face recognition. The sharpness of a face image refers to the degree of clarity in both coarse and fine details in the face region.

Many measures for computing image sharpeness have been proposed in recent years 78 . Intuitively, N.K.Ratha et al. 5 proposes a variance based method to evaluate the sharpness of biometric images. In his method, an $M \times N$ image is divided into $m \times n$ regions. In every region, the variance is computed and if it is larger than a pre-defined threshold, this region is sharpness enough and considered as a good region. The final sharpness score is derived as the ratio of numbers of good regions to the total region number.

$$
\text { Sharpness } 1=\frac{1}{N u m} \sum_{i=1}^{N u m} I\left(D_{i}>T\right)
$$

where $N u m$ is the number of regions, $D_{i}$ is the variance in region $i$ and $I(\cdot) \in$ $\{0,1\}$ is an indication function of a boolean condition.

We propose a novel method in frequency domain to evaluate the sharpness of face image. The low-frequency information in an image corresponds to the global shape of components whereas the high-frequency counterpart corresponds to the details of skin. The out of focus face images usually lose high-frequency information. Fig. 4 illustrates two examples. The left is the blurring image captured in real world and the right is the simulated image by Gaussian convolution. It can be clearly seen the high frequency coefficients of blurring images (the lower images) are really small in their frequency domain.

Enlightened by the fact that little high-spatial frequency exists in most images, DCT are widely used in image compression. In this paper we utilize DCT and IDCT to measure the high-spatial frequency content, i.e., the sharpness of an image. In particular, with an input image $I$, it is first transformed into frequency domain by DCT operation. The coefficients which occupies the predefined ratio (ratio is chosen in sequence of run-length coding according to rate,

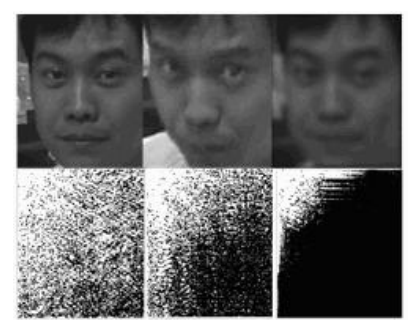

(a)

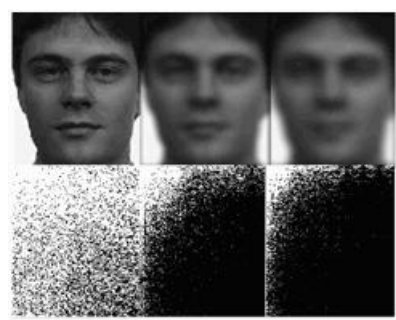

(b)

Fig. 4. Images of different sharpness degrees and their DCT frequency results 
where ratio $=M \times N \times$ rate ) of total energy is preserved and then it is inversetransformed to the image space by IDCT operation to obtain the recovered image $R$. The sharpness is finally evaluated by the difference of input image and the recovered one (Eq. 5). Obviously, the larger difference is, the sharper the input image is.

$$
\text { Sharpness } 2=\frac{1}{M \times N} \sqrt{\sum_{i=1}^{M} \sum_{j=1}^{N}(R(i, j)-I(i, j))^{2}}
$$

\section{Experiments}

The following experiments first examine on the facial asymmetry caused by non-frontal lighting and improper facial poses using the facial symmetry based methods presented in Section 2. Then DCT based method described in Section 3 are presented to measure the image sharpness.

CMU-PIE database is utilized to assess the facial symmetry problem. Images under different lighting conditions and different poses from 68 subjects are selected. In sharpness measurement experiment, 1850 images from FERET database are selected.

\subsection{Lighting Symmetry}

The proposed method is compared with the method that computes the difference on gray images directly, denoted as DDG and the LBP based method in [1. All the images are cropped according to the automatically detected eye positions.

Fig. 5 shows the cropped face images under different lighting conditions and Fig. 6] illustrates the results of different facial symmetry assessment methods. It can be seen that the DDG method, due to its sensitive to misalignment and noise, is almost infeasible in lighting symmetry evaluation. The performance of the proposed Gabor based method and the LBP based method [1] are similar and they can be used to evaluate the lighting condition better.

\subsection{Pose Symmetry}

Four poses $(27,29,11,14)$ are selected to do the pose assessment. (Fig. 7). Similarly, the proposed Gabor based method is compared with DDG and LBP method in 1]. Fig. 8 shows the face quality asymmetry values for 4 pose categories. It can be seen the Gabor and LBP based methods are more applicable.

\subsection{Sharpness Measurement}

In this section, both real and simulated images are tested. In simulated case, the blurring images are generated by the convolution operation on original images with Gaussian filters (Eq. 6).

$$
G(x, y)=\exp \left\{-\frac{x^{2}+y^{2}}{2 \sigma^{2}}\right\}
$$


Light 17
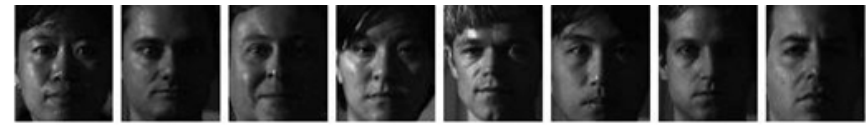

Light 15
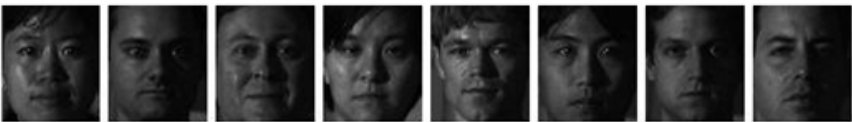

Light 13
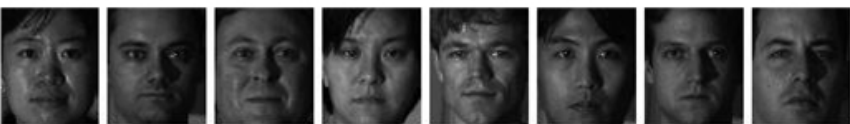

Light 11
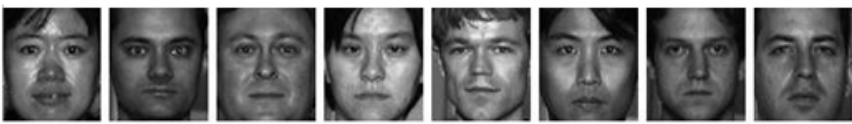

Fig. 5. Face examples under four different lighting conditions

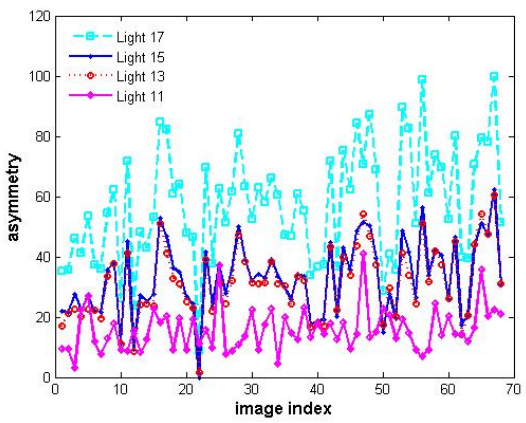

(a)

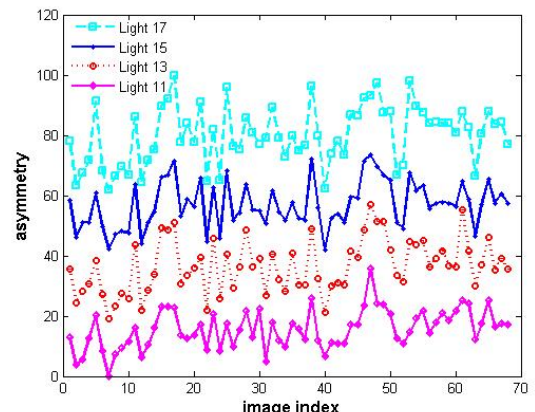

(b)

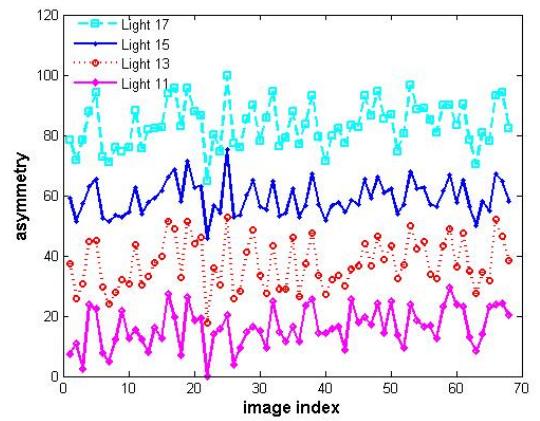

(c)

Fig. 6. Lighting asymmetry score using DDG (a), LBP (b) and Gabor (c) features

The proposed DCT based method is compared with the method in [5]. For method in [5], the image is divided into $14 \times 12$ and $7 \times 6$ respectively and the threshold is set as the mean of variances of all sub-regions. For the proposed 


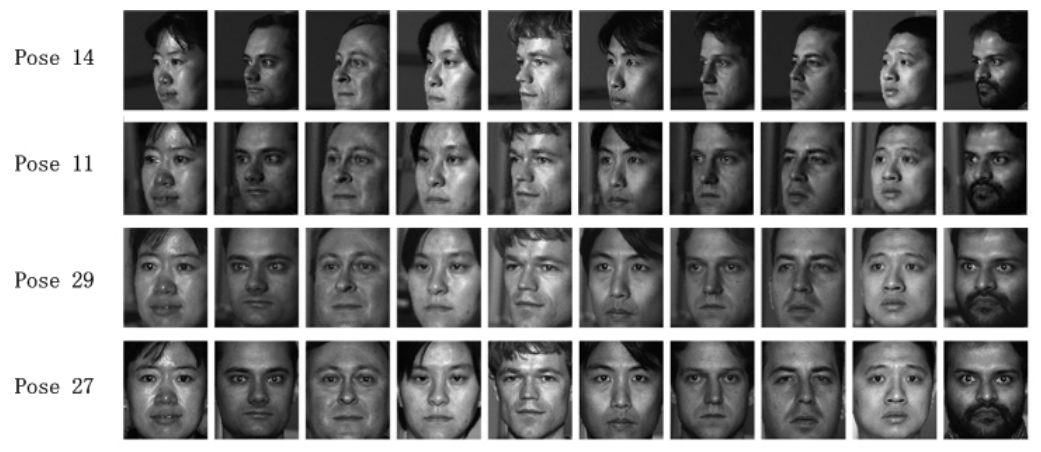

Fig. 7. Face examples under four poses

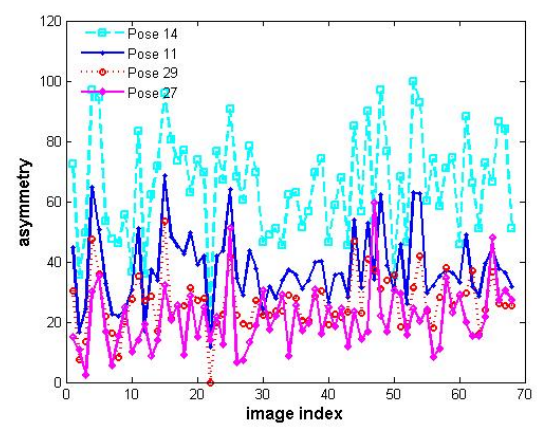

(a)

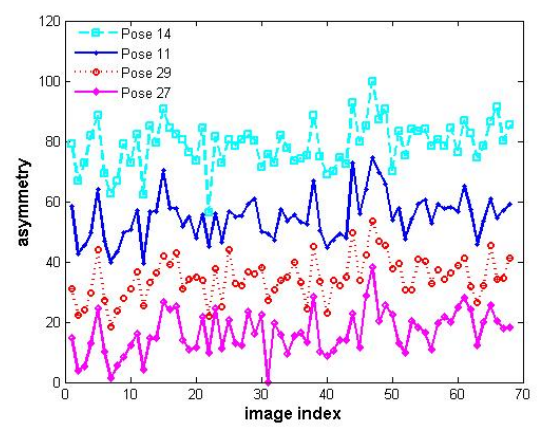

(b)

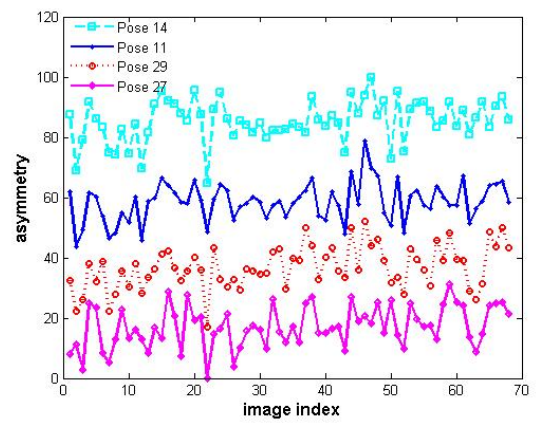

(c)

Fig. 8. Pose asymmetry score using DDG (a), LBP (b) and Gabor (c) features

method, the preserved DCT coefficient rate is set to 0.1 and 0.3 respectively. Fig. 9] illustrates the sharpness assessment results of these two methods on simulated images and real captured ones. It can be seen the proposed DCT based method achieves significantly better results to measure sharpness of images in both cases. 


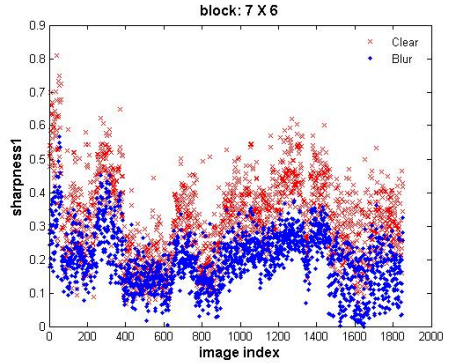

(a)

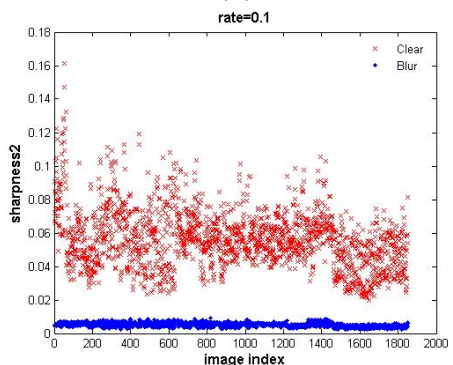

(c)

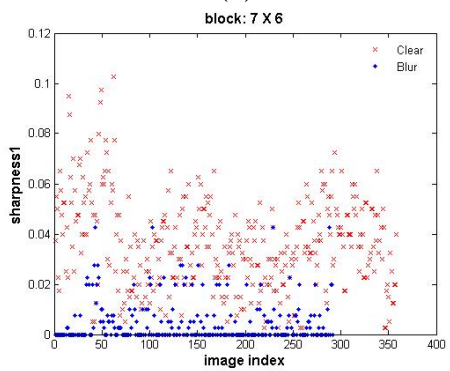

(e)

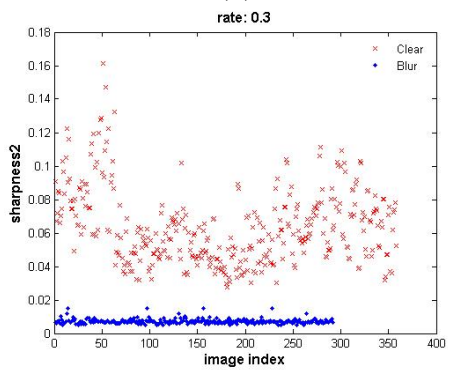

(g)

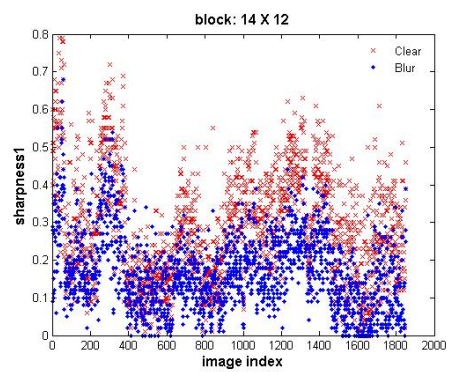

(b)

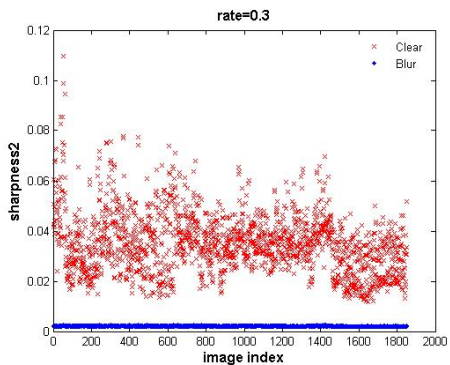

(d)

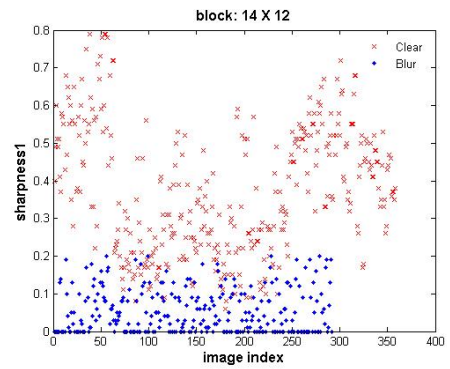

(f)

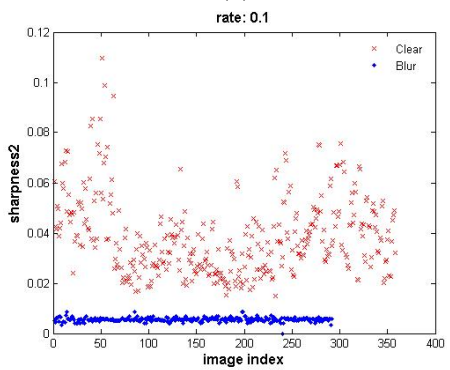

(h)

Fig. 9. Sharpness measurement results with different methods ((a)-(b) and (e)-(f) are results of the method in [5] with simulated blurring images and real captured blurring images. (c)-(d) and (g)-(h)are results of the proposed DCT method). 


\section{Conclusion}

Enlightened by draft of ISO/IEC 29794-5 where standardization of face image quality is presented, we first introduce a novel Gabor-based facial symmetry measurement method to evaluate the changing illumination and improper posture. It provides comprehension from another aspect and offers alternative choice for evaluation. As face images are cropped according to the automatically detected eye positions, situations like misalignment and anatomically non-symmetric are not excluded intentionally, which indicates stability of the method. Also a DCT and IDCT combined algorithm is proposed to discern out-of-focus, which may replenish the standardization in ISO/IEC 29794-5.

\section{Acknowledgement}

This work was supported by the following fundings: National Natural Science Foundation Project \#60518002, National Science and Technology Support Program Project \#2006BAK08B06, National Hi-Tech (863) Program Projects \#2006AA01Z192, \#2006AA01Z193, and \#2008AA01Z124, Chinese Academy of Sciences 100 People Project, and AuthenMetric R\&D Funds.

\section{References}

1. Gao, X., Li, S.Z., Liu, R., Zhang, P.: Standardization of face image sample quality. In: Lee, S.-W., Li, S.Z. (eds.) ICB 2007. LNCS, vol. 4642, pp. 242-251. Springer, Heidelberg (2007)

2. ISO/IEC JTC 1/SC 37 N 1477. Biometric Sample Quality - Part 5: Face Image Data Sample Quality (Working Draft for comment) (February 12, 2007)

3. ISO/IEC JTC 1/SC 37 N 506. Biometric Data Interchange Formats Part 5: Face Image Data (March 22, 2004)

4. Liu, C., Wechsler, H.: Gabor feature based classification using the enhanced fisher linear discriminant model for face recognition 11(4), 467-476 (2002)

5. Ratha, N.K., Chen, S.Y., Jain, A.K.: Adaptive Flow Orientation-based Feature Extraction in Fingerprint Images. Pattern Recognition 28(11), 1657-1672 (1995)

6. Engeldrum, P.G.: Psychometric Scaling: A Toolkit for Imaging Systems Development. Imcotek Press (2000)

7. Shaked, D., Tastl, I.: Sharpness measure: towards automatic image enhancement 1(1), 937-940 (2005)

8. Wee, C., Paramesran, R.: Measure of image sharpness using eigenvalues. Inf. Sci. 177(12) (2007)

9. Zhao, W., Chellappa, R., Phillips, P., Rosenfeld, A.: Face Recognition: A Literature Survey. ACM Computing Surveys 35(4), 399-458 (2003) 